\title{
Investigation of Swirling Flows in Mixing Chambers
}

\author{
Jyh Jian Chen and Chun Huei Chen \\ Department of Biomechatronics Engineering, National Ping Tung University of Science and Technology, Pingtung 912, Taiwan
}

Correspondence should be addressed to Jyh Jian Chen, chaucer@mail.npust.edu.tw

Received 9 November 2010; Revised 25 February 2011; Accepted 1 March 2011

Academic Editor: Guan Yeoh

Copyright ( $\odot 2011$ J. J. Chen and C. H. Chen. This is an open access article distributed under the Creative Commons Attribution License, which permits unrestricted use, distribution, and reproduction in any medium, provided the original work is properly cited.

\begin{abstract}
This investigation analyzed the three-dimensional momentum and mass transfer characteristics arising from multiple inlets and a single outlet in micromixing chamber. The chamber consists of a right square prism, an octagonal prism, or a cylinder. Numerical results which were presented in terms of velocity vector plots and concentration distributions indicated that the swirling flows inside the chamber dominate the mixing index. Particle trajectories were utilized to demonstrate the rotational and extensional local flows which produce steady stirring, and the configuration of colored particles at the outlet section expressed at different Re represented the mixing performance qualitatively. The combination of the Taylor dispersion and the vorticity was first introduced and made the mixing successful. The effects of various geometric parameters and Reynolds numbers on the mixing characteristics were investigated. An optimal design of the cylindrical chamber with 4 inlets can be found. At larger Reynolds number, Re $>15$, more inertia caused the powerful swirling flows in the chamber, and more damping effect on diffusion was diminished, which then increased the mixing performance.
\end{abstract}

\section{Introduction}

In the last decades, microfluidic devices have been widely utilized in microelectromechanical systems and employed as powerful instruments to perform biological experiments. Some products have been mass-produced, such as micropump, micromixer, microvalve, and even microreactor. Microfluidic devices have been developed for a broad range of applications. These applications cover genomic analysis $[1,2]$, chemical engineering [3], as well as lab-on-a-chip [4]. Effective mixing of various fluids is required in many miniaturized multicomponent flow systems. Fast mixing can reduce the time required for analysis and improve reaction efficiency in industrial applications. Micromixer can be integrated in a microfluidic system or used as a single device. Furthermore, the investigation of micromixers is the basis for understanding the transport phenomena in micro-scale systems. Traditionally, both the stirring and the creation of turbulent flow are exploited to improve the mixing characteristics in the macroscopic world. However, it is very difficult to create the conventional mixing mechanisms inside the miniaturized systems because the Reynolds number is rarely larger than 100 .
In general, since the Reynolds number is very low in a microscopic system (Reynolds number, Re, indicates the ratio of inertia to viscous forces and is expressed as $\operatorname{Re}=$ $U D_{H} / \nu$, with $U$ being the inlet flow velocity, $D_{H}$ the hydraulic diameter of the microchannel, and $v$ the kinematic viscosity), liquid flows inside microchannels are generally observed as laminar flows. Viscous forces dominate the flow fields, and vortices cannot exist in such flow. As a result of the small geometric sizes and the laminar flow regimes in microfluidic analytical devices, fast mixing cannot be achieved by the conventional methods. In a typical microfluidic device, the mixing of two or more miscible fluid streams is dominated by molecular diffusion, which is driven by a concentration difference and is a rather slow process. According to Fick's first law of diffusion, the flux of the diffusing species is proportional to the diffusivity and a divergence of the concentration [5]. In addition, the mixing time increases in proportion to the square value of the diffusing distance and furthermore depends on the diffusivity of the diffusing compound [6]. In order to speed up mixing, the essentials of a diffusion-based micromixer are, therefore, the maximization of the contact area of different fluids and the minimization of the diffusing distance. Among these 


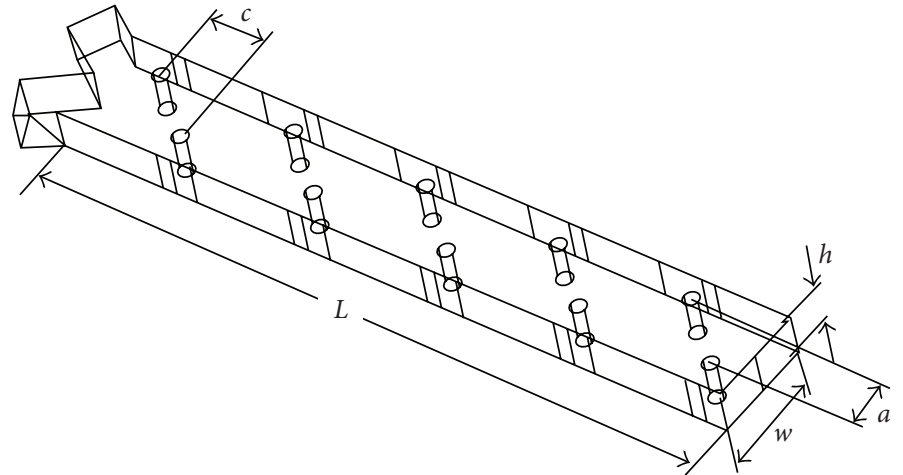

(a)

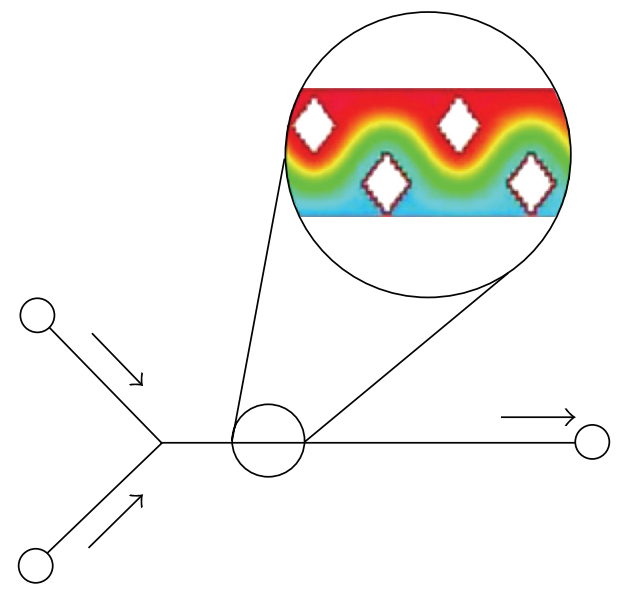

(c)

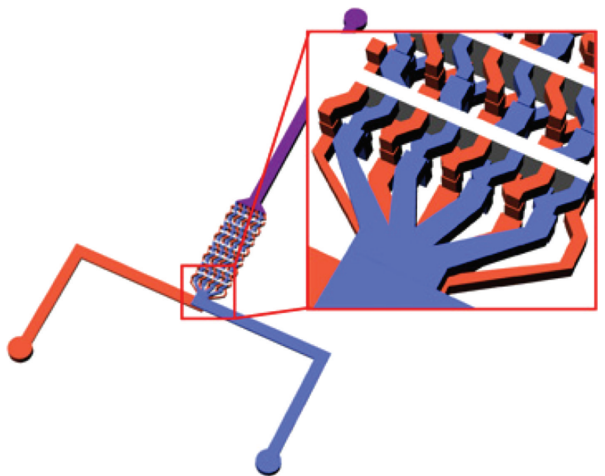

(b)

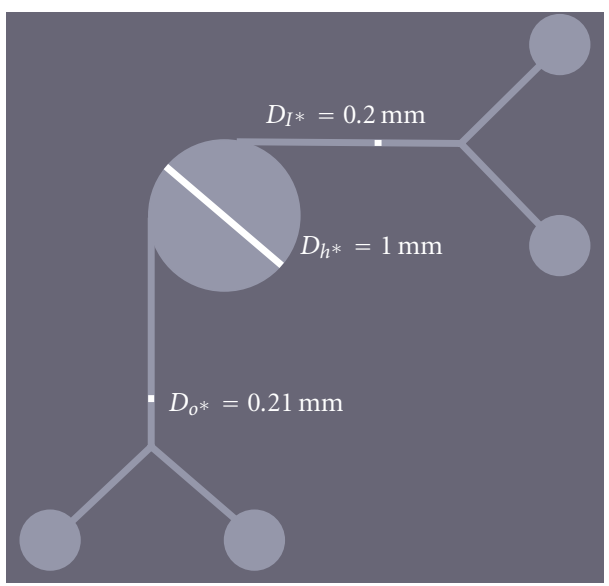

(d)

Figure 1: A few representative designs of the parallel laminated micromixers recently developed by other research. (a) Maeng et al. [14], (b) Cha et al. [17], (c) Bhagat et al. [18], and (d) Long et al. [26].

technologies a number of micromixers have been developed. These can be classified into two groups: active and passive micromixers. Though the time and channel length required for active mixing are less than those for passive mixing, the active micromixers are difficult to fabricate, clean, and integrate into microfluidic systems. The pronounced advantage of passive micromixers is that they utilize no external power except the mechanism used to drive the fluids into the microfluidic systems.

During the past ten years, studies on passive mixing in micromixers have been conducted. Nguyen and Wu [7] presented an elaborate review for the micromixers and reported on the progress of recent development of micromixers. Since generating a turbulent liquid flow inside the microdevice is complicated, the passive micromixers primarily have two types of mixing mechanisms, namely, chaotic advection and molecular diffusion. A chaotic advection micromixer refers to unit operation that stretches and folds fluid volumes over a channel cross-section $[8,9]$. The challenges of chaotic micromixers are the microfabrication of complex structures. By considering the characteristics of molecular diffusion, some have dealt with the injection mixing, where one liquid fluid is injected into the other with microplumes [10]. Some studies proposed micromixers based on the increase of the boundary surface, with the concept of lamination [11]. These designs split incoming streams into several narrower confluent streams and rejoined these narrower streams together. Then the contact surface of two fluids is implemented, and it increases the diffusion speed. The thickness of each fluid layer is greatly reduced in comparison with the characteristic diffusion length, which reduces the mixing time.

Parallel laminated mixers with simple two-dimensional structures were fabricated without difficulty, and mixing in such laminar flows can be very easily enhanced. In all of the aforementioned studies, two representative micromixers were discussed in detail. One design is a device with multiple intersecting channels. A micromixer based on the principle of distributive mixing was presented by Bessoth et al. [12], and the feasibility of the chip being used for mixing in the millisecond regime was demonstrated. Hinsmann et al. [13] also introduced a micromixing device. The fluid entering through the inlet was split and forced to flow under the separating layer. Then the fluid streams entered the main channel and were mixed. To improve the mixing by placing obstacles in the channel shown in Figure 1(a), Maeng et al. [14] created the disruption to the flow field, which reduced the diffusion path. An in-plane micromixer using the Coanda 
effect was designed by Hong et al. [15], and a Poisseuille flow was produced in the perpendicular direction of the flow to achieve a great mixing performance. A passive micromixer using a recycle flow was devised by Jeon et al. [16], and a recycle flow was introduced from side channels to the inlet flow. Cha et al. [17] proposed a mixer designed for chessboard mixing shown in Figure 1(b), and the mixer achieved a high mixing performance in a very short channel length by enlarging the contact areas between two liquid fluids. Bhagat et al. [18] reported a planar passive mixer shown in Figure 1(c), and this design incorporated diamondshaped obstructions within the channel to breakup and recombine the flows.

The other design split the main streams into several narrower streams and rejoined them together. A laminated micromixer was first reported by Koch et al. [19] who conducted two silicon-based mixers to achieve good mixing performances. The mixer geometries, which share an interdigital arrangement of inlet streams, were proposed by some researchers $[20,21]$. Results suggested that geometric focusing of a large number of liquid streams is a powerful micromixing principle. In order to increase the mixing by reducing the diffusion distance, the main stream is needed to be split into several substreams. In the case of the mixer utilizing distributive mixing, the pressure loss is higher because of the narrower intersecting channels. A circular vortex micromixer with sixteen tangential inlets was presented by Bohm et al. [22]. The liquids were injected into the micromixer to induce a swirling flow field, and the mixing could be performed in a shorter time-scale. Lin et al. [23] developed a three-layer glass vortex mixer for low Re. Two inlet channels divide into 8 individual channels tangent to a circular chamber. The Re is higher than a value of 2.32, and a self-rotation effect is induced in the chamber. A swirl micromixer with two branch channels, a central chamber, and an exit tube was carried out by Jin et al. [24]. The dependence of the mixture viscosity and density on the mass fraction of solution was taken into consideration. The cost-effective mixing at Re $>500$ was obtained because of the generation of the swirling flow. Yang et al. [25] presented a vortex-type micromixer with four tangential inlets which utilized pneumatically driven membranes to generate a swirling flow. A mixing efficiency of 0.95 could be achieved in time period of $0.6 \mathrm{~s}$ for the twomembrane layout. A three-dimensional polystyrene vortex micromixer was demonstrated by Long et al. [26] and shown in Figure 1(d). The device employs one inlet, one vertical cylinder, and one outlet. Results were compared with the mixing performance of a two-dimensional square-wave channel.

For the vortex micromixer, many substreams are needed to improve the mixing since the mixing of two or more fluid streams occurs by virtue of molecular diffusion. To reduce the pressure and increase the contact area of two fluids, the mixing chamber inside the micromixer is imposed. In previous work $[22,23]$, eight or sixteen inlets were used to inject two different kinds of fluids into the mixing chamber. The more inlets utilized, the more significantly swirling effects could be seen. However, the experimental apparatus

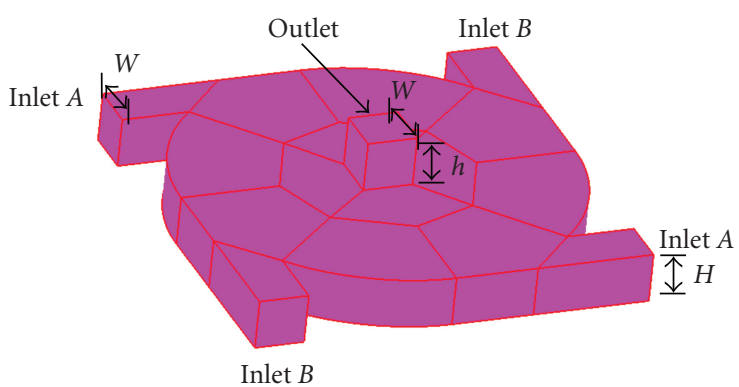

Figure 2: One of the basic configurations for the analysis. The diameter of the cylindrical chamber is equal to $800 \mu \mathrm{m}$. The width, $W$, and height, $H$, of the inlets are set at the value of $100 \mu \mathrm{m}$. The width, length, and height of the outlet are set at the value of $100 \mu \mathrm{m}$.

would be more complicated in order to supply all these inlet flows. The effects of the numbers of the inlet channels have not been studied in detail. In all of the aforementioned studies, the effects of mixer geometries on flow characteristics inside the vortex micromixers were not investigated. The geometric effects of the mixer affect the liquid flows, so the success of the mixing depends strongly on this effect. In fluid dynamics, vorticity is considered as the circulation per unit area at a point in a fluid flow field. Besides, whereas two fluids are flowing in a flat channel, both convective and molecular diffusions contribute to mixing performance due to the Taylor dispersion effect. The combination of the radial dispersion and the vortex flow makes the mixing successful, and no previous studies examined the flow and mixing performances inside the vortex micromixer by utilizing the concepts of the vorticity and the Taylor dispersion. Characterization of the mixing performance of mixing devices using CFD simulations has been described in many publications. The particle trajectories are used to numerically study the mixing and fluidic behaviors by chaotic advection inside the microchannel. Therefore, understanding the fluid flows in vortex micromixer by utilizing the particle trajectories is of marked importance in the related fields of microfluidics.

The objective of the present study is to demonstrate a designed lamination micromixer with a right square prism, an octagonal prism, or a cylinder, plus several pairs of inlets and one outlet. A three-dimensional fluid field is used to describe the flow characteristics in the microfluidic system. The effects of swirling flows on mixing performances are expressed in terms of particle trajectories, vorticity profiles, concentration distributions, and mixing indexes. The effects of various geometric parameters and $\mathrm{Re}$ on the mixing characteristics were also investigated.

\section{Mathematical Model}

Figure 2 schematically depicts one of the basic configurations for the analysis. The physical problem is that the liquids to be mixed enter the mixing chamber at a specified speed from several tangential inlets and then flow out from the outlet channel at the central location of the chamber. The physical domain is a three-dimensional volume consisting of a right 
square prism, an octagonal prism, or a cylinder. $W$ is the width of the inlet channel, and $H$ is the height of the inlet channel. In addition, $h$ is the height of the outlet channel having the length and the width of $L$. The boundary surfaces of the domain are solid, rigid, and impermeable walls. It is assumed that the steady state is reached in the chamber and the channels; the variations of the concentration do not modify the viscosity and the density of the fluid; the channel walls are assumed to be smooth. The effects of surface tension on the interaction between two miscible liquids on Earth are masked by the effects of gravity and density. On Earth, miscible liquids effectively combine into one relatively homogenous (or equally distributed) solution [27]. There is no real interface when liquids are perfectly miscible. Surface tension forces in this study are neglected.

The equations to be solved are the continuity equation, the Navier-Stokes equations, and the conservation of species equations, along with the appropriate boundary conditions. The following assumptions have been adopted to obtain a proper mathematical formulation of this problem.

(1) Fluid flow is Newtonian and laminar. As reasonably assumed herein, the fluid is incompressible since the velocities encountered in this type of flow are expected to be markedly lower than the sonic velocity in the fluid.

(2) All thermophysical properties of the liquid are to be evaluated at the fixed reference temperature of $300 \mathrm{~K}$.

(3) Gravity is negligible.

(4) The nonslip and nonpenetration conditions are usually encountered at solid surfaces.

The conservation of mass and momentum equations are solved to determine the flow field of the liquids. In symbolic notation, the continuity equation can be expressed as follows:

$$
\nabla \cdot \vec{U}=0
$$

where $\vec{U}$ is the fluid velocity vector.

The momentum equation for a continuum is the analogue of Newton's second law for a point mass. In symbolic notation the momentum equation is expressed as

$$
\rho \vec{U} \cdot \nabla \vec{U}=-\nabla P+\mu \nabla^{2} \vec{U}
$$

where $\rho$ is the fluid density, $P$ is the pressure, and $\mu$ is the fluid viscosity.

Species transport by pressure-driven flows occurs as a result of convection and diffusion and can be described by the combined species convection-diffusion equation. In symbolic notation the diffusion-convection equation is

$$
\rho \vec{U} \cdot \nabla \phi=D \nabla^{2} \phi
$$

where $D$ is the diffusivity and $\phi$ is the mass concentration. This equation must be solved together with (1) and (2) in order to achieve computational coupling between the velocity field solution and the concentration distribution.
The liquids enter into the mixing chamber from several tangential inlets and then flow out from the outlet channel at the central location of the chamber. The rotation of a fluid about a vertical axis can be observed in the chamber. Reynolds number in the physical domain, Re, indicates the ratio of inertial forces due to rotation of a fluid about a vertical axis to viscous forces. The effects of inertia on the elastic instabilities in Dean and Taylor-Couette flows were investigated through a linear stability analysis [28]. It was shown that, when rotation of the inner cylinder drives Taylor-Couette flow, the Reynolds stresses produce energy, and thus are destabilizing, while for the flow driven by the rotation of the outer cylinder alone, the Reynolds stresses dissipate energy, thus stabilizing the flow. The stability of axially symmetric flow at nonzero Reynolds number was also analyzed [29]. The effect of inertia on axisymmetric disturbances was investigated and showed that inertia tended to destabilize the flow. The physical system in our micromixer is that the liquids enter the mixing chamber with a fixed wall from several tangential inlets and then flow out from the outlet channel at the central location of the chamber. So inertia force tends to destabilize a system, and then the mixing performance can be enhanced. On the other hand, viscous force tends to stabilize a system and damp out perturbations, such as the diffusion.

\section{Numerical Analysis}

For a better understanding of the fluid flow and the mixing characteristics in the mixer, a computational fluid dynamics package, $\mathrm{CFD}-\mathrm{ACE}+$, using a finite volume approach is utilized to simulate three-dimensional flow fields as well as two-fluid mixing. A nonlinear steady-state algorithm is used for hydrodynamic calculations, and a linear steady-state one is applied to solve the diffusion-convection equation. The inlet boundary condition is set to have the same constant velocity for two fluids, and the outlet is assumed to be a constant pressure condition. The user scalar module enables us to compute the transport of species. In this study, the transport mechanism in fluid volumes is assumed, and species do not affect the velocity, or any other computed field. For the scalars, the boundary conditions are generalized as:

$$
\alpha\left(D \frac{\partial \phi}{\partial n}\right)_{B}+b \phi_{B}=c .
$$

For the Dirichlet boundary conditions at the inlet, $a$ is set to $0, b$ is set to 1 , and $c$ is set to 1 (for species A) or 0 (for species B).

This study considered three different configurations of the flow system. The general configuration consists of a right square prism, an octagonal prism, and a cylinder (c.f. Figure 3). Three dimensional structured grids are employed. The grid systems of the computation domain are chosen to assure the orthogonality, smoothness, and low aspect ratios to prevent the numerical divergence. Poor grid systems can enhance the numerical diffusion effects. Numerical errors due to discretization of the convective terms in the transport equation of the concentration fields introduce an 


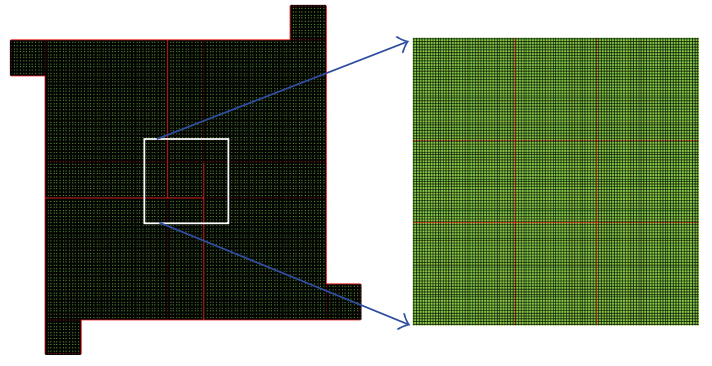

(a)

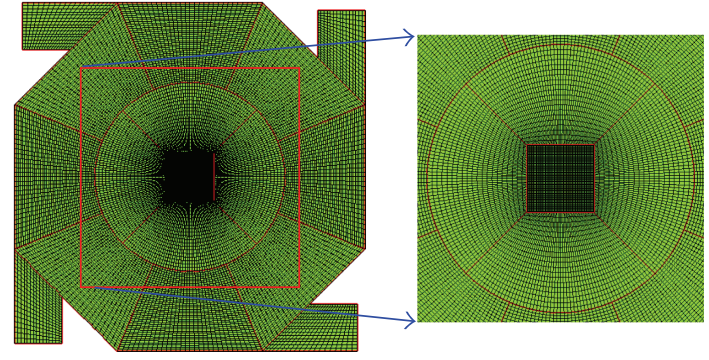

(b)

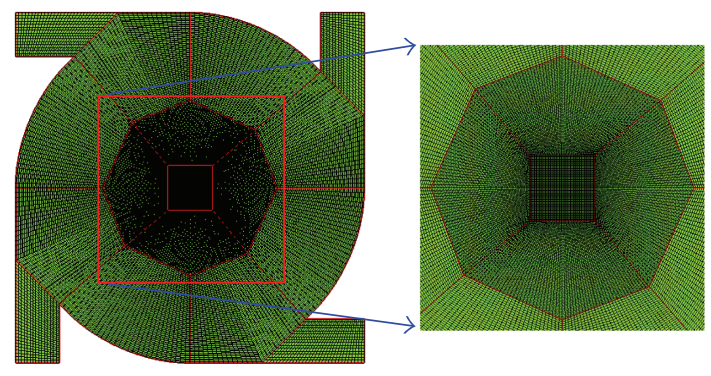

(c)

Figure 3: Grid systems of three configurations: (a) right square prism, (b) octagonal prism, and (c) cylinder.

additional, unphysical diffusion mechanism [20]. This socalled numerical diffusion is likely to dominate diffusive mass transfer on computational grids. Higher-order discretization schemes reduce the numerical errors. Numerical diffusion strongly depends on the relative orientation of flow velocity and grid cells, and it can be minimized by choosing grid cells with edges parallel to the local flow velocity. Regarding to our grid systems shown in Figure 2, the numerical diffusion can be reduced comprehensively in the computational results. To minimize the effects of the meshing on mixing, the mesh density is increased until the mixing index variation is less that $5 \%$.

The SIMPLEC method is adopted for pressure-velocity coupling, and then all spatial discretizations are performed using the second-order upwind scheme. The algebraic multigrid (AMG) solver is utilized for pressure correction, whereas the conjugates gradient squared method (CGS) and preconditioning (Pre) solver are employed for velocity and species corrections. The solution is considered to be converged when the relative errors of all independent variables are less than $10^{-4}$ between the sweeps $n$ and $n+1$.

The cross-sectional images of outlets obtained from the CFD software are converted to text formats. The uniformity of mixing at sampled sections is assessed by determining the mixing index $\varphi$ of the solute concentration, which is defined as

$$
\varphi=1-\frac{\int_{A}\left|I-I_{\mathrm{ave}}\right| d A}{\int_{A_{0}}\left|I_{0}-I_{\mathrm{ave}}\right| d A},
$$

where I is the concentration value (between 0 and 1) on the sampled section $A, I_{0}$ is the concentration value at the inlet plane $\mathrm{A}_{0}$, and $I_{\text {ave }}$ is the averaged value of the concentration over the sampled section. The mixing index $\varphi$ range is from 0 for no mixing to 1 for complete mixing.

\section{Conceptual Design}

In this section, the design concept of a three-dimensional swirling micromixer is introduced. From the preliminary studies on the vortex micromixers, a strongly rotational motion of the fluids is generated. A designed lamination micromixer with a chamber, plus several pairs of inlets and one outlet, is demonstrated. The diameter of the cylindrical chamber is equal to $800 \mu \mathrm{m}$. The inlets are tangential to the chamber, and the outlet is located in the central region of the chamber. The width, $\mathrm{W}$, and height, $\mathrm{H}$, of the inlets are set at the value of $100 \mu \mathrm{m}$. The width, length, and height of the outlet are set at the value of $100 \mu \mathrm{m}$, too. All the inlets are symmetric with the central axis of the mixing chamber. The fluid properties are set to the physical and thermodynamic properties of water, which are diffusivity, $D$, of $1 \times 10^{-9} \mathrm{~m}^{2} / \mathrm{s}$, kinematic viscosity, $v$, of $1 \times 10^{-6} \mathrm{~m}^{2} / \mathrm{s}$, density, $\rho$, of $1 \times 10^{3} \mathrm{~kg} / \mathrm{m}^{3}$. The fluid with red color stands for species A, and the fluid with blue color for species $\mathrm{B}$. The concentration of species is normalized as one and zero for the inlet of species A and species B, respectively. The parameters of the above values are used, unless noted otherwise.

A streak line is defined as a line formed by the particles which pass through a given location in the flow field [30]. In a steady flow, the streak lines coincide with the stream lines and the particle trajectories. These streak lines can be seen as the trajectories of the particles released from the specific locations of the inlets to the outlet and used to describe the nature of the flow field inside the micromixer. Figure 4 depicts the streak lines as the fluids flow through 


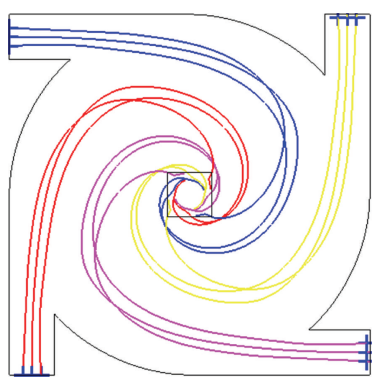

(a)

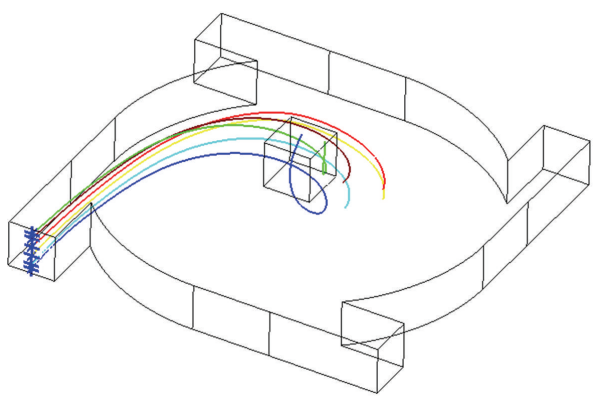

(c)

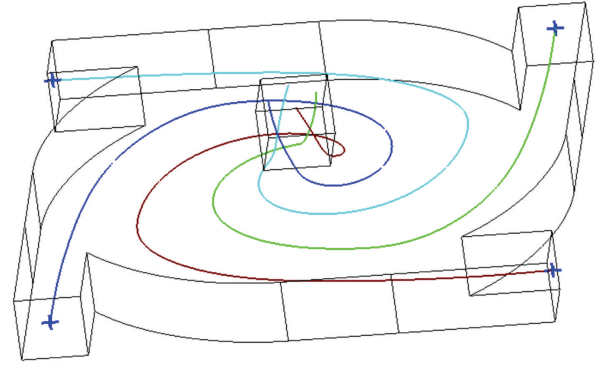

(b)

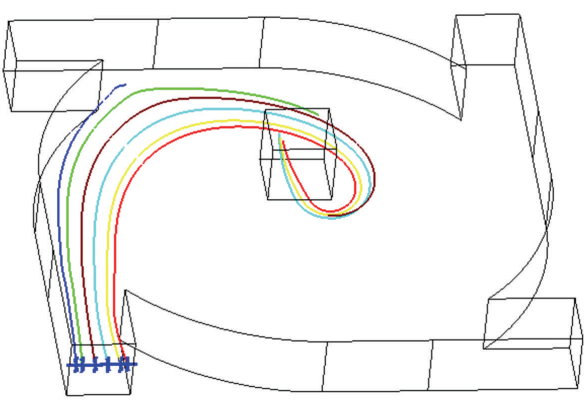

(d)

Figure 4: The trajectories of the microfluidic flow in the mixer. (a) Top view of the streak lines in the micromixer. (b) Four streak lines starting from the central locations of the four inlets. (c) Six streak lines starting from the six specific locations along vertical central line of one inlet. (d) Six streak lines starting from the six specific locations along horizontal central line of one inlet.

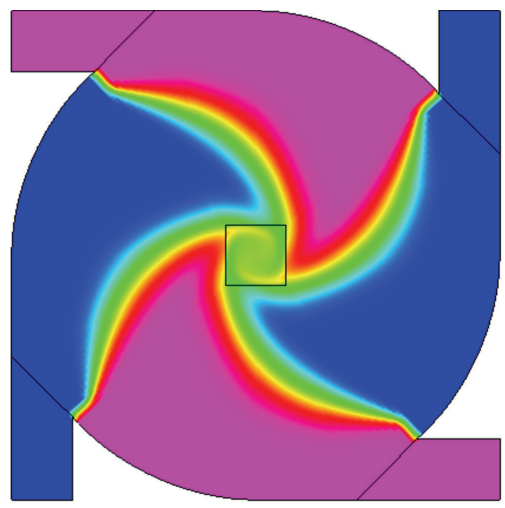

(a)

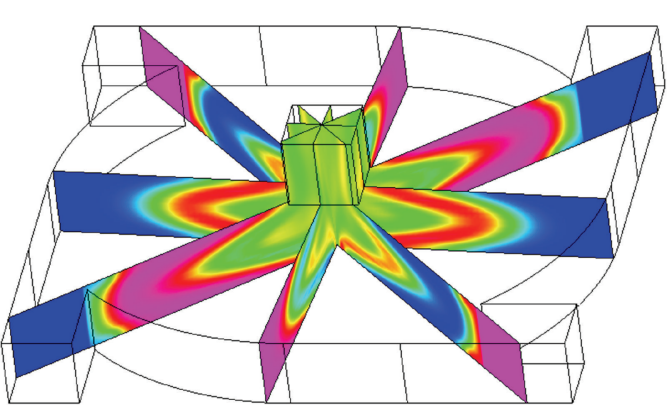

(b)

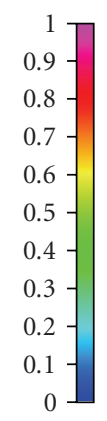

FIGURE 5: Numerical mixing results of mixer with cylinder (a) from the top view and (b) at 8 cross-sectional areas. the cylindrical chamber at the inlet velocity of $0.75 \mathrm{~m} / \mathrm{s}$ and the chamber height of $100 \mu \mathrm{m}$. The corresponding Re is 75 . The initial positions of particles are shown as markers "+" illustrated in the figures. Fluids enter the chamber in the tangential directions, and the flow is almost unidirectional. As the fluid approaches the entrance of the other fluid, the fluid is deflected and forms a clockwise vortex (Figure 4(a)). These streak lines are symmetric with the center of the chamber. The rotational flow field is observed and extends towards the outlet of the mixer (Figure 4(b)). As shown in Figures 4(c) and 4(d), the trajectories are quite regular.
Thus the stretching of the interface can be presented, and the rotational and extensional local flow can produce steady stirring in the chamber.

In order to perform a comprehensive analysis of the mass transfer mechanism in vortex micromixer, the crosssectional concentration distributions are utilized to demonstrate the mixing characteristics. The concentration distributions for mixing in the cylindrical chamber are shown in Figure 5. The predicted concentration distribution of two fluids regarded from the top and eight cross-sectional areas are presented in Figures 5(a) and 5(b), respectively. In order 


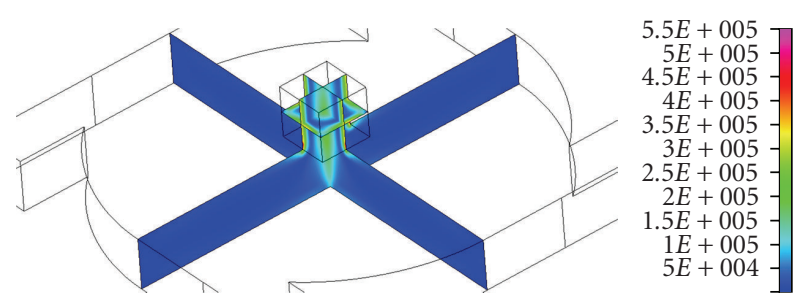

(a)

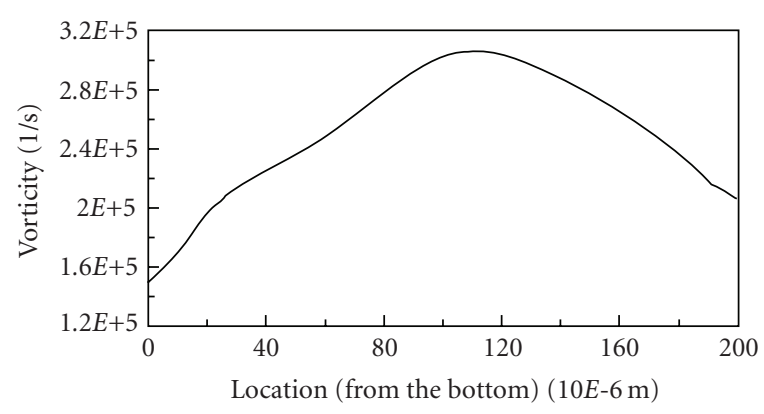

(b)

FIguRE 6: (a) The vorticity distributions at three cross-sectional areas of the chamber with cylinder and (b) numerical results of vorticity profiles along the central line of the mixer.

to improve the mixing, two different fluids enter into the four inlets alternately. The inlet channel is tangential to the mixing chamber, and a rotation flow field is induced owing to the high inertia force, as shown in Figure 5(a). The fluid, A, meets the other fluid, B, near the inlet B and the interface of two fluids becomes curved. The contact area of two fluids is increased. Then two fluids enter into the outlet channel. Because the area of the outlet is much smaller than that of the chamber, the diffusion distance of two fluids in the outlet channel becomes smaller and the mixing can be enhanced. Two fluids flow tangentially into the chamber and flow clockwise inside the chamber. Since the outlet is located at the central region of the chamber, fluids tend to flow out of the outlet channel and the vortex flow is formed. Flows in the angular and radial directions are both significant. Whereas two fluids are flowing in a capillary tube, both convective and molecular diffusions contribute to mixing performance due to the axial dispersion effect. This is the so-called Taylor dispersion. In this micromixer, the height of the chamber is small, and the shear force establishes the radial concentration gradient enhancing the mass transport rate in the radial direction. The radial dispersion effect can be observed in Figure 5(b). This system shows the mixing characteristics similar to Taylor dispersion, with contributions from both diffusion and convection. The combination of the radial dispersion and the vortex flow makes the mixing successful. In fluid dynamics, vorticity is the curl of the fluid velocity. It can also be considered as the circulation per unit area at a point in a fluid flow field. It is a vector quantity whose direction is along the axis of the fluid's rotation. So the value of the vorticity inside the chamber volume can be seen as the indicator of the vortex flow. Figure 6(a) shows the vorticity distributions at three cross-sectional areas of the chamber with cylinder, the same as the case illustrated in Figure 4. It is presented that the vorticity is much larger near the central region of the chamber and the outlet channel than in other regions. So the rotation rate of the fluid is greatest at the center and decreases progressively with distance from the center. The vorticity greatly depends on the velocity gradient in the mixer. The fluids enter into the outlet channel, and the gradient of the velocity near the wall of the channel becomes larger; then the vorticity increases more. As shown in Figure 6(b), the vorticity along the central axis from the bottom of the chamber increases, reaches the maximum value near the entrance of the outlet channel, and then decreases to the location of the outlet. The vortex flow is intensified more near the entrance of the outlet channel; the contact areas of fluids can be further enlarged.

In an effort to understand the two-fluid mixing inside the mixer, the velocity vector planes are utilized to demonstrate the rotation of the fluid flows. Figure 7 illustrates the mixing and flow characteristics at eight cross-sectional areas from the bottom of the chamber for the case presented in Figure 4. The location of the cross-sectional area in Figure 7(a) is $25 \mu \mathrm{m}$ from the bottom of the chamber. The distance between the following, from Figures 7(b) to 7(h), are $25 \mu \mathrm{m}$ apart. The concentration distributions and the vector planes are shown. With respect to the mass transport mechanism in mixing, two fluids flow tangentially into the chamber and the radial component of velocity grows because fluids tend to flow through the outlet channel. These two components result in an overall rotating flow field inside the chamber. The fluids flow clockwise, and the contact area between them increases as shown in Figure 7(a). In the central region of the chamber, and the mixing performance is great. The rotation effect grows as the fluids move away from the bottom of the chamber. The mixing region spreads from the central region, and the mixing becomes much better, as presented in Figure 7(b). Whereas the fluids approach the top of the chamber, the mixing region shrinks owing to the no-slip conditions at the wall of the chamber. However, the rotation effect increases, as shown in Figure $7(\mathrm{c})$, the mixing is enhanced even more. At the location of the upper wall of the chamber, the pattern of the mixing characteristics, as shown in Figure 7(d), is similar to the pattern in Figure 7(a). The circulation of the fluid flow in the chamber is clearly observed. The maximum velocity is also shown near the central region of the chamber. Then the fluids enter into the channel of the outlet. The distance of molecular diffusion is reduced much more as the fluids flow from the chamber into the channel. Thus the rotating flow achieves superior mixing. The increased interface area of two fluids can promote a mass transfer based on diffusion. The configurations of interfacial areas between two different fluids play an important role 

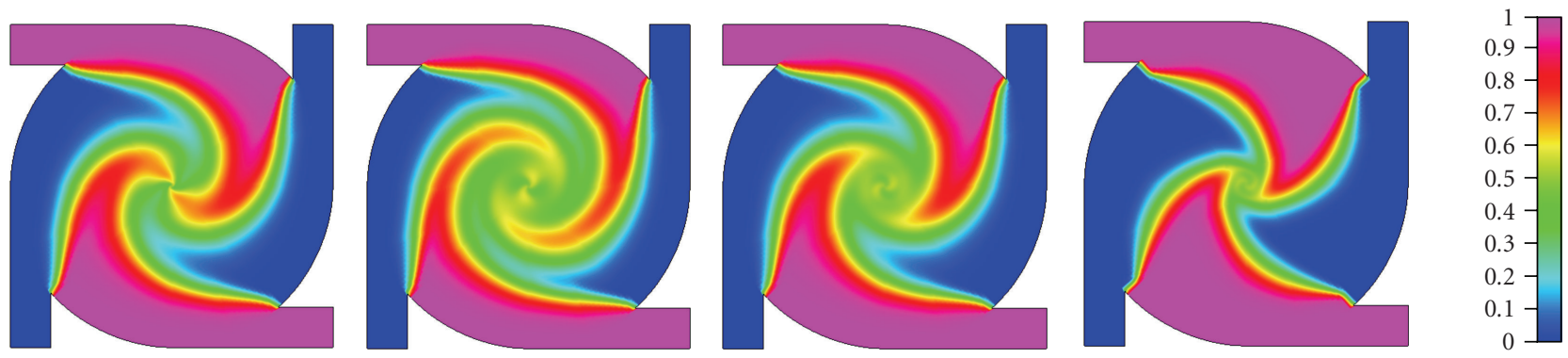

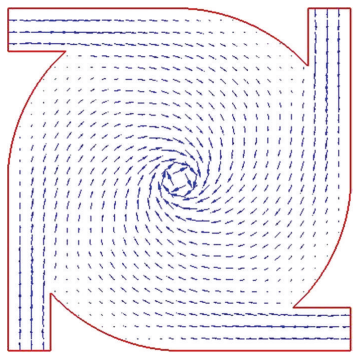

(a)

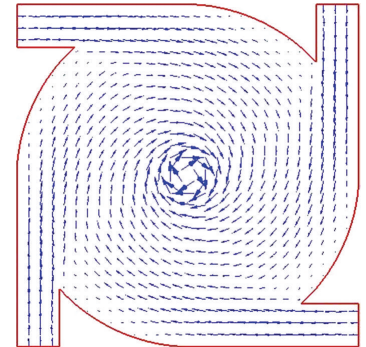

(b)

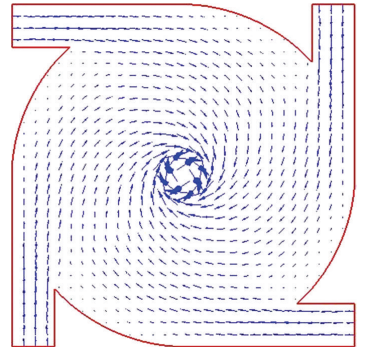

(c)

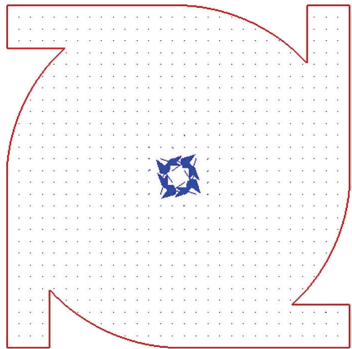

(d)
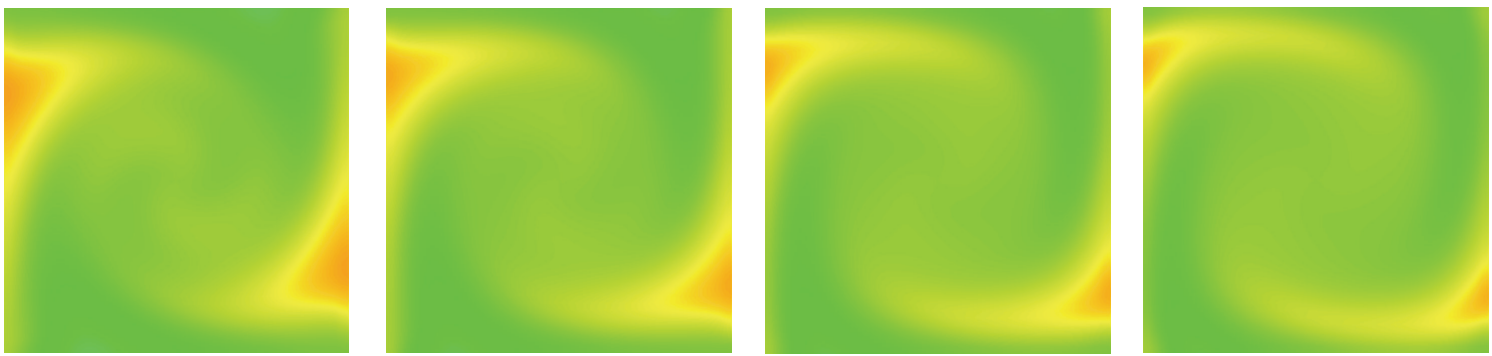

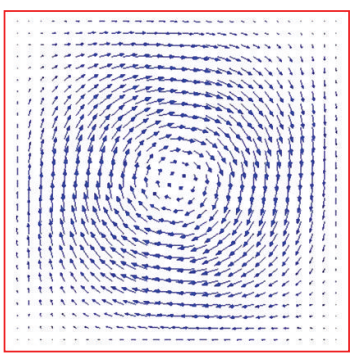

(e)

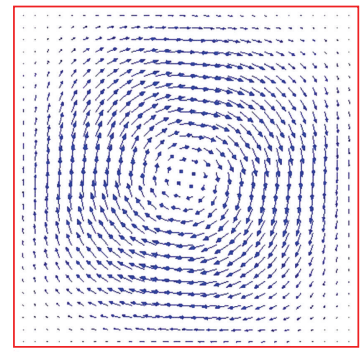

(f)

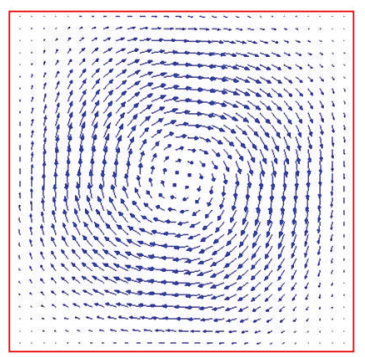

(g)

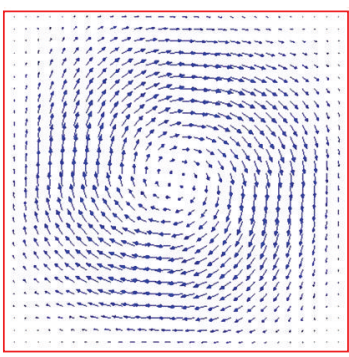

(h)

FIgURE 7: The concentration distributions and the vector planes at eight cross-sectional areas from the bottom of the chamber. (a) $25 \mu \mathrm{m}$, (b) $50 \mu \mathrm{m}$, (c) $75 \mu \mathrm{m}$, (d) $100 \mu \mathrm{m}$, (e) $125 \mu \mathrm{m}$, (f) $150 \mu \mathrm{m}$, (g) $175 \mu \mathrm{m}$, and (h) $200 \mu \mathrm{m}$ from the bottom of the chamber.

in the micromixers. As shown in Figure 8, the interfacial regions of two fluids are demonstrated. The interfacial regions represent the area in which the concentration over the sampled section is equal to the value of the concentration equal to 0.5 . Due to the cylindrical chamber of the mixer, as the case shown in Figure 4, the interfacial area between two fluids was stretched. Four inlets and one outlet in the mixer are studied to show the influences of self-rotation effects; it is found that interfacial layers are stretched spirally from the intersections of the inlet channels and the chamber to the central region of the chamber. The interfacial regions between two fluids are enlarged, and steady stirring flow is created, which are exploited to enhance the mixing performances.

\section{Results and Discussion}

This study solved the concentration distributions of liquid fluids in the micromixers, and the effects of various geometric parameters on momentum and mass transports in 


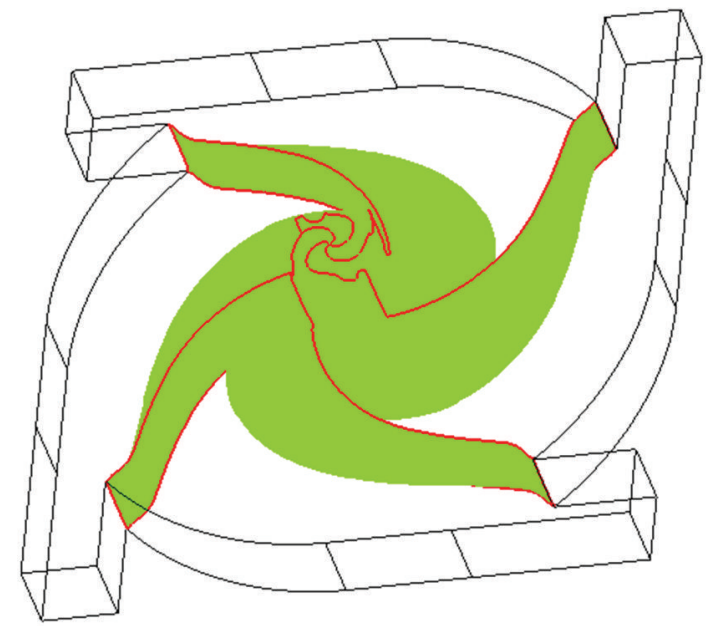

FIGURE 8: Interfacial regions of the concentration equal to 0.5 .

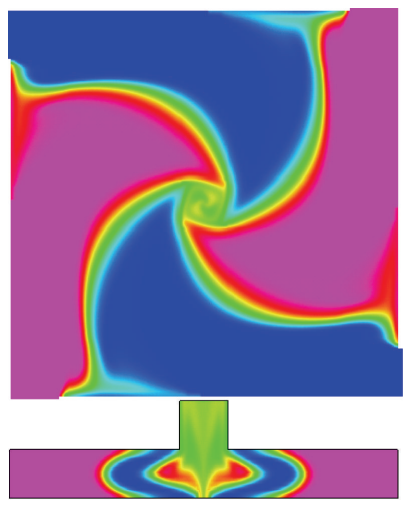

(a)

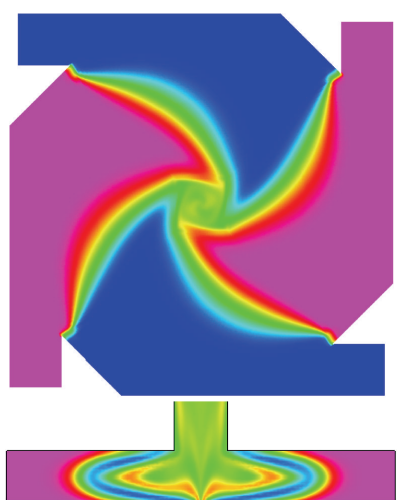

(b)
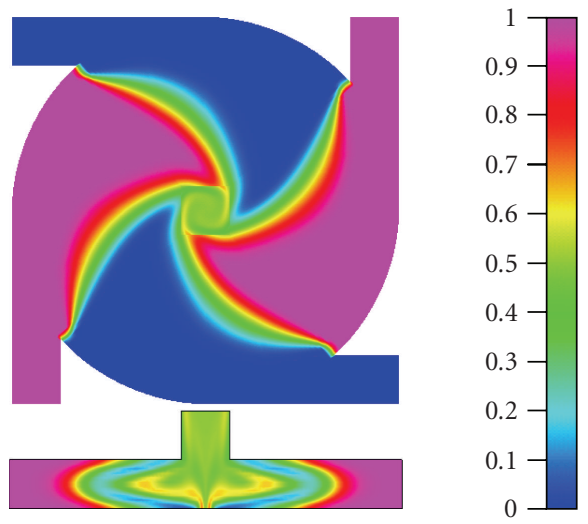

(c)

FIGURE 9: The results of mixing with various configurations: (a) right square prism, (b) octagonal prism, and (c) cylinder.

solution were examined. It also investigated the influences of various Reynolds numbers on the momentum and mass transfer characteristics in the fluids in terms of the mixing index and concentration distributions. In the following micromixers with different geometric chambers which are right square prism, octagonal prism, and cylinder with four inlets and one outlet are studied to show the influences of self-rotation effects on mixing.

The following is a comparison of the transfer of momentum and mass in mixing chambers with different configurations and examining the influences of the various geometrical parameters on mixing results. The length of the right square prism is equal to $800 \mu \mathrm{m}$. The length of each side of the octagonal prism is about $200.63 \mu \mathrm{m}$, and the distance between two opposite sides of the octagonal prism is equal to $800 \mu \mathrm{m}$. The diameter of the cylinder is equal to $800 \mu \mathrm{m}$. Figure 9 summarizes the results of mixing with various configurations. The velocity of all inlets is equal to $0.75 \mathrm{~m} / \mathrm{s}$, and the height of the mixing chamber, $h$, is $100 \mu \mathrm{m}$, as well as the height of the inlet channel. The predicted concentration profiles of two fluids regarded from the top and the cross-section are presented. Corresponding results are listed from left to right for chambers which are right square prism, octagonal prism, and cylinder, respectively. The corresponding mixing index of the outlet is $0.881,0.877$, and 0.893 . The radial dispersion in the chamber is affected by the configurations of the mixing chamber. In Figure 9(a), the interface of two fluids can be clearly observed. The effect of the radial dispersion is the smallest for the case of right square prism. From the results shown in Figure 9(c), the mixed fluids are well dispersed near the central region of the chamber, and the effect of the radial dispersion is the largest for the cylinder. The vortex flow is intensified more for the cylindrical chamber where the contact areas of fluids can be further enlarged. Results show that the self-rotation effect is enhanced in micromixers with cylindrical chamber, which makes this mixer an effective vortex mixer.

To perform the higher mixing index, various numbers of inlets are used to investigate the mixing characteristics of micromixers with the chambers of octagonal prism, as 

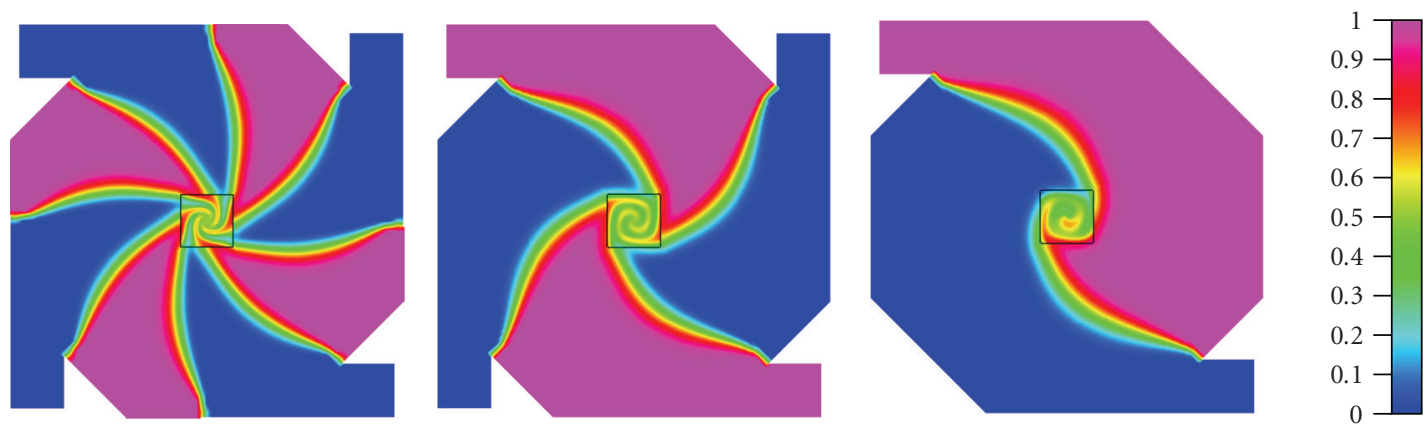

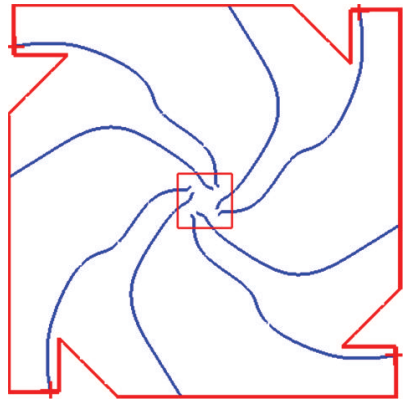

(a)

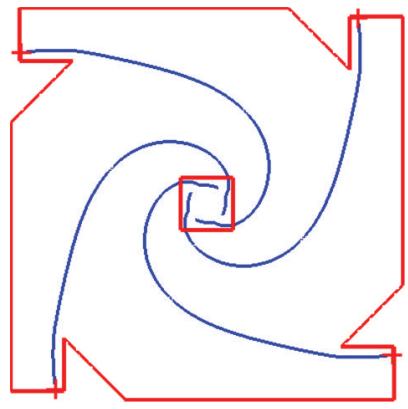

(b)

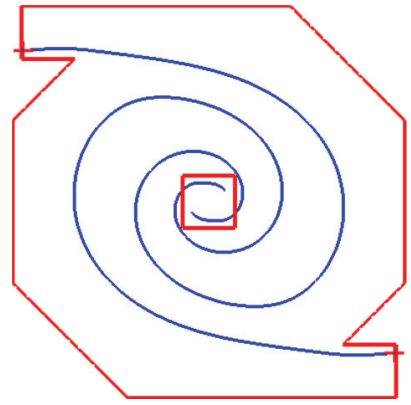

(c)

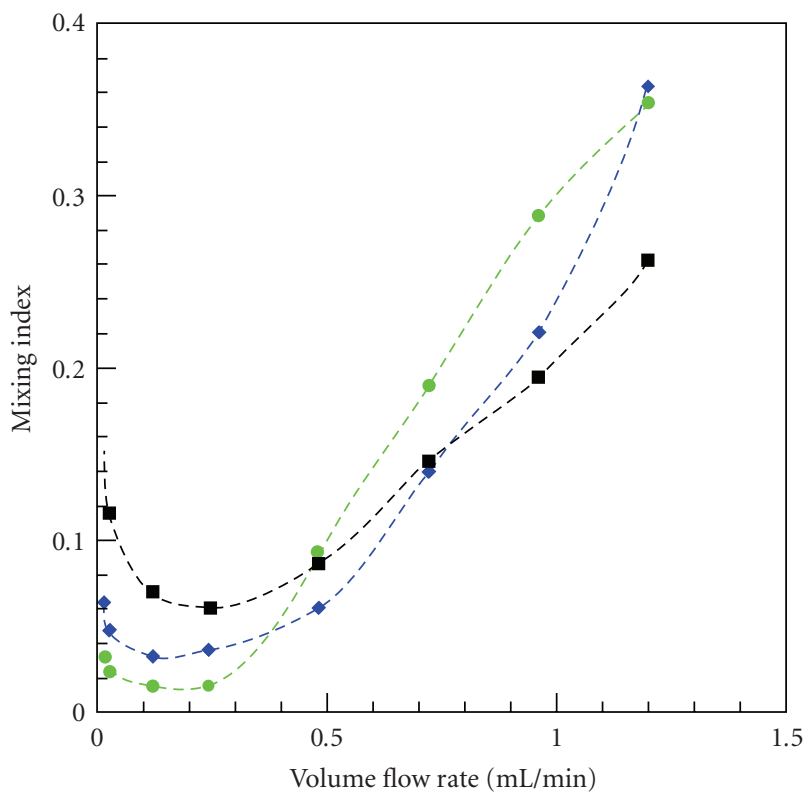

- Present work $\square$

- Jin et al. [25] O

- Lin et al. [24]

(d)

FIGURE 10: The results of concentration distributions and streak lines with various numbers of inlet channels: (a) 8, (b) 4, and (c) 2. (d) Comparison results with the previous study $[23,24]$.

shown in Figure 10. Results of 8, 4, and 2 inlets with the inlet velocity, equal to $0.25 \mathrm{~m} / \mathrm{s}, 0.5 \mathrm{~m} / \mathrm{s}$, and $1 \mathrm{~m} / \mathrm{s}$, respectively, are presented in Figures 10(a), 10(b), and 10(c), respectively. For the number of inlets equal to 8 , the contact areas of two fluids increase dramatically. The shape of the cross-sectional area of the outlet is square, and 8 inlets are used in the mixer. It is found that the pattern of the mixing characteristics is not symmetric with respect to the center of the chamber. 


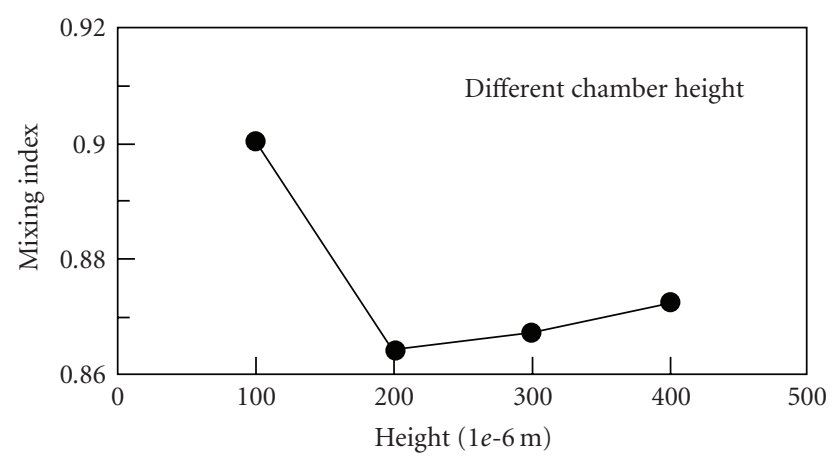

(a)

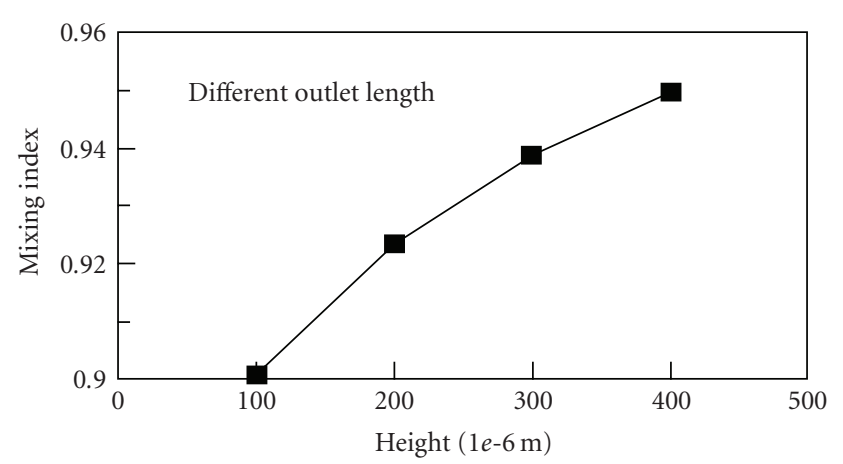

(b)

Figure 11: The effects of chamber heights and the length of the outlet channel on mixing.

As the red fluid (species A) approaches the entrance of the blue fluid (species B), it is deflected and forms a clockwise vortex. Then it flows into the entrance of the outlet channel. The fluid of species B meets the fluid of species A around the joint of the inlet and the chamber, where it is deflected and also forms a clockwise vortex. However, the inlet velocity is small $(0.25 \mathrm{~m} / \mathrm{s})$, and it enters the outlet channel directly. The swirling effects are shown in Figure 10(a). Figure 10 also depicts the streak lines as the fluids flow through the chamber with octagonal prism. It is shown in Figure 10(a) that the trajectories of species $B$ are quite similar to the cases shown in Figures $10(\mathrm{~b})$ and 10 (c). However, the trajectories of species $\mathrm{A}$ are different from the trajectories of species B. For the case of 4 inlets, the contact areas of two fluids decrease in the chamber. Due to the large inlet velocity $(0.5 \mathrm{~m} / \mathrm{s})$, it swirls around the central region at a longer path and it is the result of the large mixing region that is created. For the case of 2 inlets, the contact areas of two fluids decrease significantly, and the interfacial layer only exists from the joint of the inlet of species A and the chamber to the other joint along the diagonal line. The corresponding mixing index for the chambers with 8,4 , and 2 inlets is $0.676,0.785$, and 0.681 , respectively. The mixing chamber with 4 inlets shows the greatest mixing index among these micromixers. So an optimal design of the chamber with 4 inlets can be found. This study analyzes three different configurations of a microfluidic system via numerical simulations. The results are compared with the previous works [23, 24]: lamination micromixers with a cylinder, plus one or four pairs of inlets and one outlet. Results of various total inlet flow rates on the mixing performance are presented in Figure 10(d). Because the inertial force of the fluids was so small that it is negligible at flow rates less than $0.5 \mathrm{~mL} / \mathrm{min}$, mixing is dominated by pure molecular diffusion. The interface between streams is large for the circular vortex micromixer with 8 tangential inlets. One can see that mixing in this micromixer is the highest. When the flow rate is greater than 0.5 , the increased inertial force enlarges the rotational flow. The mixing in the vortex micromixer with 2 tangential inlets is improved. Our designed mixer with 4 inlets can perform well for the range of flow rate from 0 to $1.2 \mathrm{~mL} / \mathrm{min}$.

The effects of the chamber heights, $H$, and the outlet channel lengths, $h$, on mixing in the cylindrical chamber are presented in Figure 11. The predicted mixing index at various chamber heights (upper portion) and at various channel lengths (bottom portion) is shown. Corresponding results are listed from (1) to (4) for chamber height equal to $100 \mu \mathrm{m}, 200 \mu \mathrm{m}, 300 \mu \mathrm{m}$, and $400 \mu \mathrm{m}$, respectively, with the length of outlet channel being $100 \mu \mathrm{m}$. And corresponding results are listed from (5) to (8) for channel length equal to $100 \mu \mathrm{m}, 200 \mu \mathrm{m}, 300 \mu \mathrm{m}$, and $400 \mu \mathrm{m}$, respectively, with the height of chamber being $100 \mu \mathrm{m}$. The mixing index of the chamber decreases at the start and then increases slowly because the vortex flow is weak for the cases with larger chamber length. Nevertheless, the contact areas in the chamber with larger volume are augmented. The mixing can be improved, and the mixing index starts to increase for the cases (3) and (4). Whereas two fluids enter into the outlet channel, the axial flow of fluids is dominant and the rotation of the fluids is almost constant along the channel. The contact areas are enlarged with the increasing channel length; then the mixing index improves as well.

Figure 12 plots the effects of various inlet velocities on the mixing for the height of the mixing chamber being $100 \mu \mathrm{m}$. They show not only the mixing within the microchamber, but also the vortex index of the mixer. The inertial force is very important when the inlet velocity is $1 \mathrm{~m} / \mathrm{s}$ and the corresponding Re is 100 . Because the wall of the inlet channel is tangential to the wall of the chamber and the inertial force drives the liquid flow forward, the fluid meets the other fluid with strong inertia. The large inertia force causes the fluid to proceed fast and for a large clockwise vortex to form. Inertia force tends to destabilize a system, whereas viscous force tends to stabilize a system and damp out perturbations, such as the diffusion. From the definition of $\mathrm{Re}$, strong inertia causes the powerful vortex flow in the chamber, and the damping effect on diffusion is diminished. The mixing performance can be enhanced. Re decreases with the decreasing inlet velocity, and the viscous force becomes dominant. The inertial effect is not very obvious, and the area of mixing region on the top wall is decreased. The inertial force is very trivial when the inlet velocity is $0.1 \mathrm{~m} / \mathrm{s}$. The competition between inertia and viscous force permits the shape of the interface to be clearly observed. It is extremely apparent that the mixing index is the lowest. Because the mixing at a position depends on the upstream 


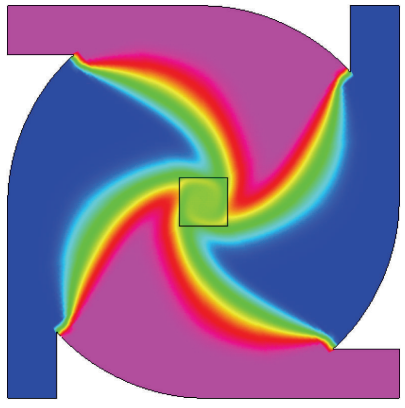

(a)

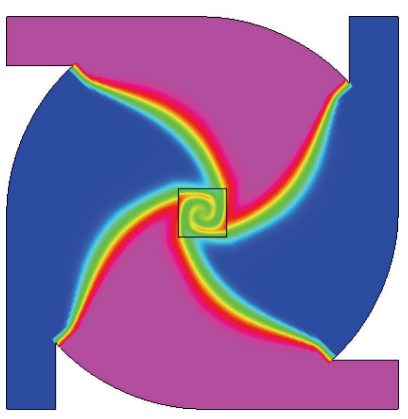

(b)

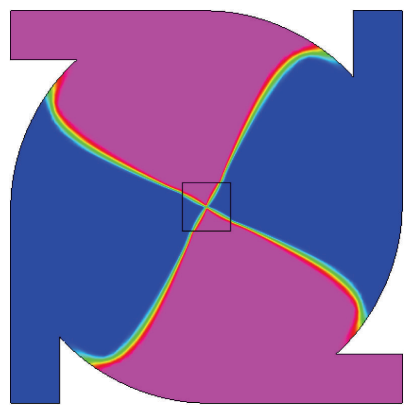

(c)

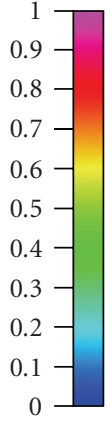

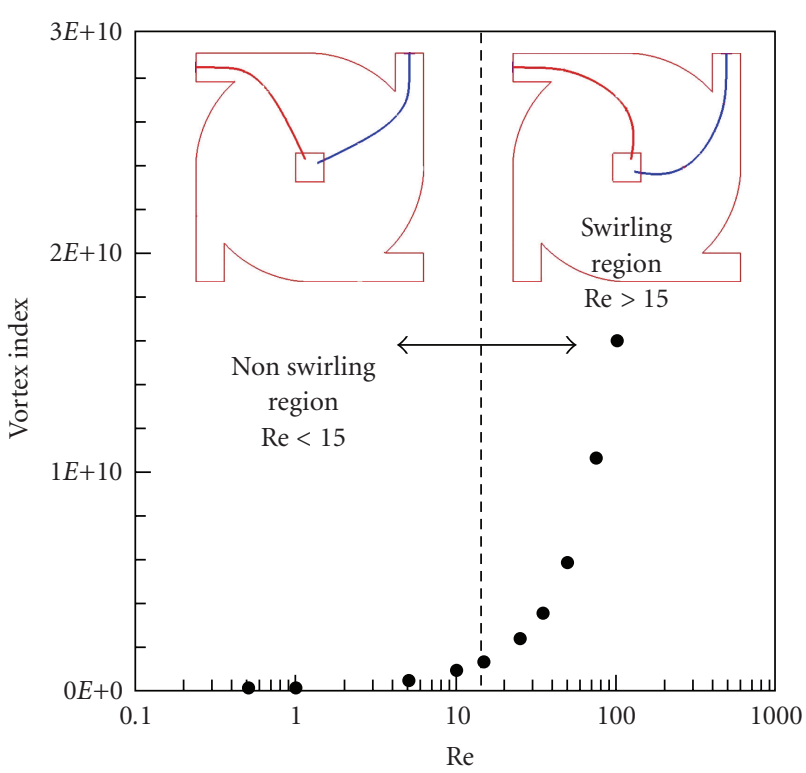

(d)

Figure 12: The results of mixing with various inlet velocities: (a) $1 \mathrm{~m} / \mathrm{s}$, (b) $0.5 / \mathrm{m} / \mathrm{s}$, and (c) $0.1 \mathrm{~m} / \mathrm{s}$. (d) The profile of the vortex index inside the chamber.

history of the flow, the vorticity history determines the mixing performance at a cross-section of the channel. A vortex index represents the vorticity history of a flow [14] and is introduced into our study. The influences of inlet velocity on vortex index are plotted in Figure 12(d). A large inlet velocity represents a large Re. At low $\mathrm{Re}, 0.5<\operatorname{Re}<15$, viscous forces in the fluid are larger than inertial forces; thus, inertia can be neglected. The streak lines from the center of the two inlets shown in the upper of Figure 12(d) almost direct towards the outlet. No swirling flow can be observed. This is the so-called no swirling region. When the Re are 15-100, both viscous and inertial forces are important. The streak lines shown in the upper right side of Figure 12(d) are deflected. The rotational and extensional flow can be created in the chamber, and the interface between the two fluids increases. Therefore the mixing performance is also improved.

The particle trajectories for the micromixer at various inlet velocities are investigated. The particle trajectories are the traces where the particles are released from the specific locations of the inlets to the outlet. In Figure 13(a), particles are released from the four inlet sections viewed from the inlet. Three lines which are located at the central line of the cross section, at the offsets of central line of 25 and $35 \mu \mathrm{m}$, are selected. Twenty-four particles per line are chosen. The configuration of colored particles at the outlet section can represent the mixing performance qualitatively and expressed at Re of 100, 50, and 10 in Figures 13(b), $13(\mathrm{c})$, and $13(\mathrm{~d})$, respectively. Particles are labeled with a specific color. The particle with red color stands for species A and the particle with blue color for species B. From the particle distributions, it is easily understood that the selfrotational effect is very advanced at an Re of 100 and the pattern of the particle distribution is very irregular. Particles with different colors are spread over the entire cross section. Then the mixing can be enhanced. Whereas the inlet velocity decreases to the value of $0.5 \mathrm{~m} / \mathrm{s}$, corresponding to an Re of 50 , four spiral-like patterns are created at the outlet cross section. Obviously, the stretched flows of the micromixer in the angular direction can be seen, and the interface of the two 


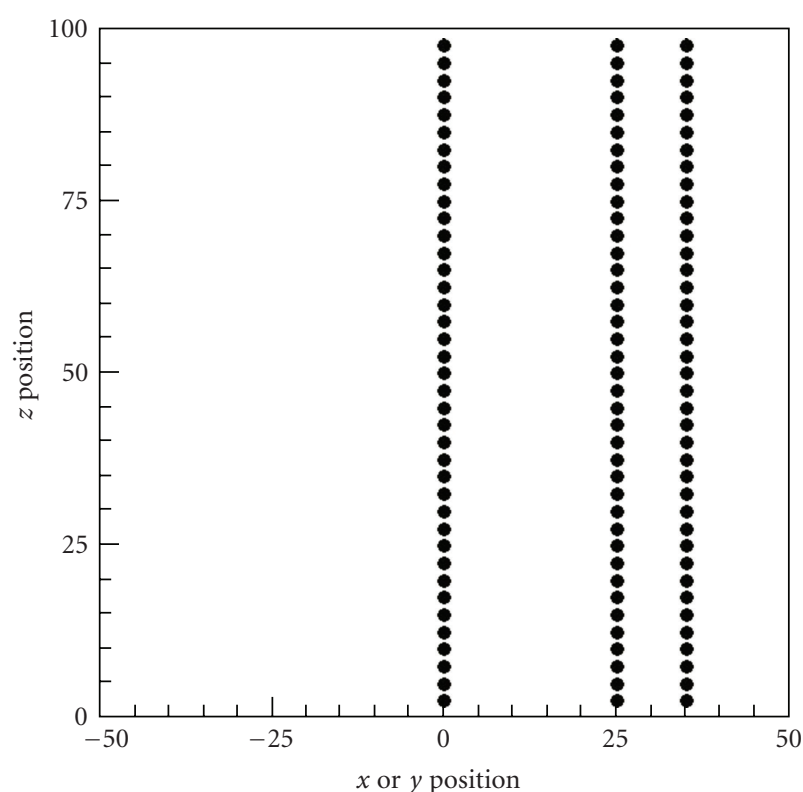

(a)

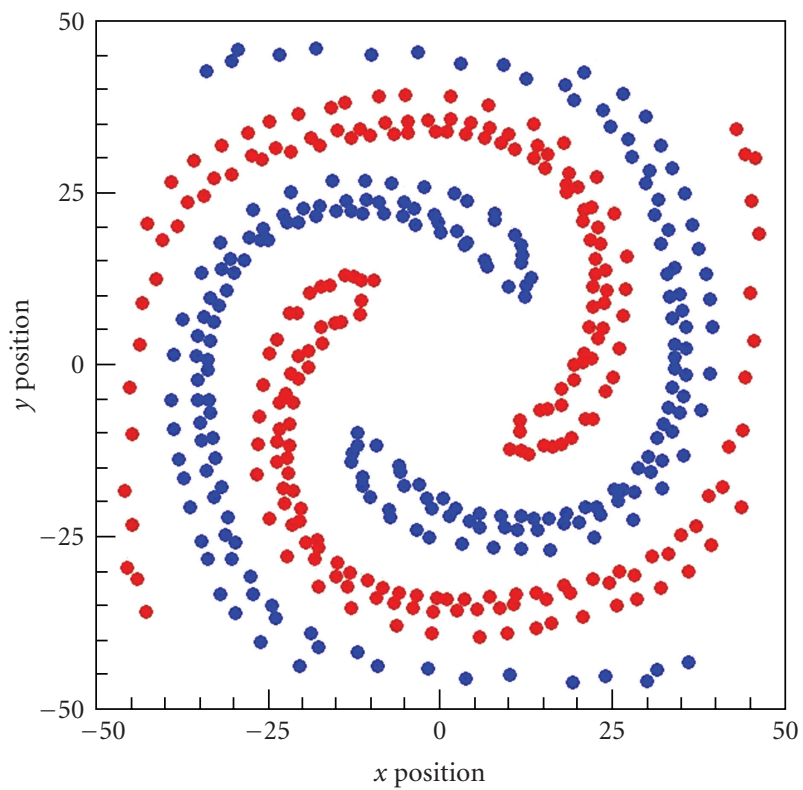

(c)

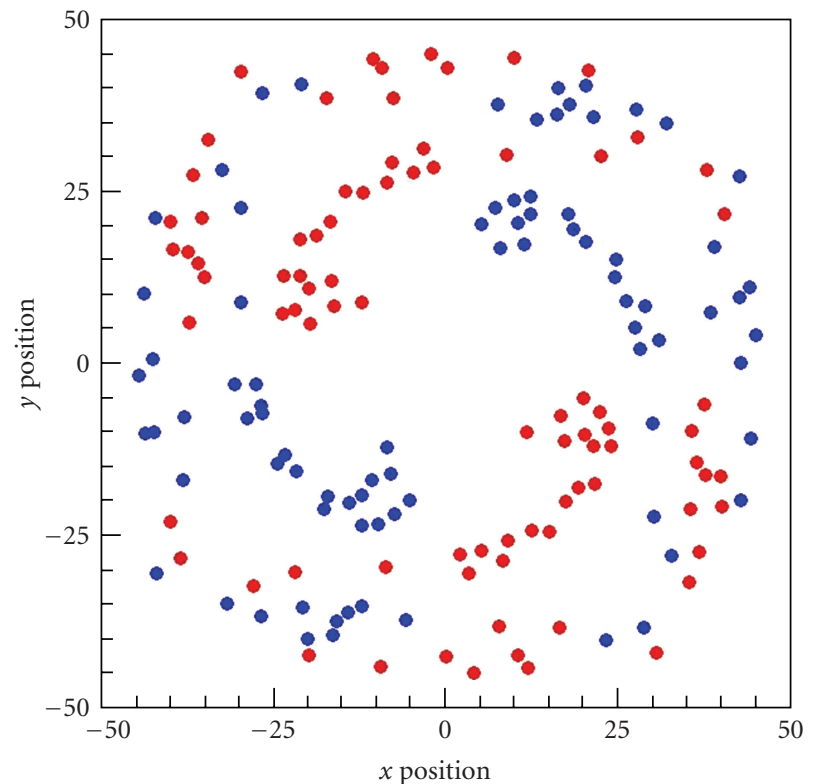

(b)

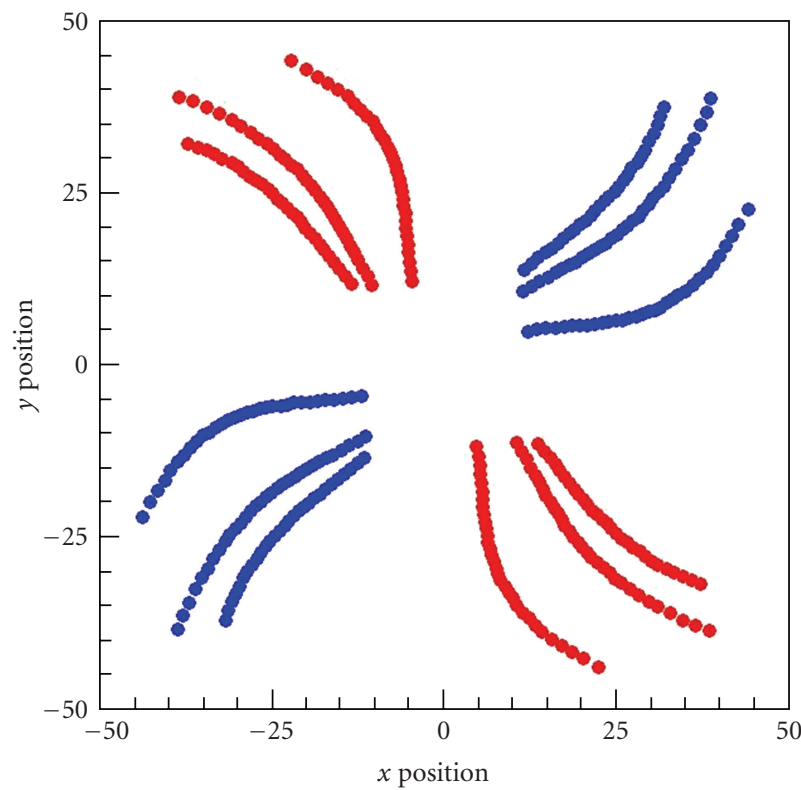

(d)

Figure 13: (a) Particles are released from the four inlet sections viewed from the inlet. Three lines which are located at the central line of the cross section, at the offset of lines of 25 and $35 \mu \mathrm{m}$, are selected. Twenty-four particles per line are chosen. The configuration of colored particles at the outlet section at Re of (b) 100, (c) 50, and (d) 10.

fluids is enhanced. These clockwise patterns are more orderly than these of an Re of 100. And a similar pattern can be seen at the outlet section in Figure 12(b). No rotational effect is observed at a Re of 10 (shown in Figure 13(d)). The patterns of the particle distributions are very simple and like straight lines. The exchange of two fluids is negligible, and the mixing is very poor.

\section{Conclusions}

This study investigated the steady behavior of threedimensional momentum and mass transfer in micro chamber which consists of a right square prism, an octagonal prism, or a cylinder. The research was studied by a computational fluid dynamics software using a finite element 
approach. Results which were presented in terms of particle trajectories, vorticity profiles, and concentration distributions indicated that the swirling flows inside the chamber dominate the mixing index. Particle trajectories were utilized to demonstrate the rotational and extensional local flows which produce steady stirring, and the combination of the radial dispersion and the vortex flow makes the mixing successful. The effects of various geometric parameters and inlet velocities on momentum and mass transfer mechanisms were discussed in detail. In addition, numbers of inlets significantly affect mixing characteristics. The mixing index decreases by using fewer inlets. With larger numbers of inlets, the significant swirling effects could be seen, and thus, the mixing index is higher. It is also shown that the force driven by the inertia has a crucial impact on the mixing. At higher Reynolds number, $\operatorname{Re}>15$, inertia causes more vortex flow in the chamber and diminishes the damping effect on diffusion, thereby leading to a higher mixing performance. Finally the configurations of colored particles at the outlet section were applied to qualitatively predict the performance of micromixers. Particular emphasis was placed on a qualitative analysis of the physical phenomena, which will benefit the study of microfluidic components in industrial applications.

\section{Acknowledgments}

The authors would like to thank the National Science Council of the Republic of China for financially supporting this research under Contract no. NSC 96-2221-E-020-021. We are also grateful to the National Center for High-performance Computing for computer time and facilities.

\section{References}

[1] M. K. McQuain, K. Seale, J. Peek et al., "Chaotic mixer improves microarray hybridization," Analytical Biochemistry, vol. 325, no. 2, pp. 215-226, 2004.

[2] S. R. Nugen, P. J. Asiello, J. T. Connelly, and A. J. Baeumner, "PMMA biosensor for nucleic acids with integrated mixer and electrochemical detection," Biosensors and Bioelectronics, vol. 24, no. 8, pp. 2428-2433, 2009.

[3] C. Rosenfeld, C. Serra, C. Brochon, V. Hessel, and G. Hadziioannou, "Use of micromixers to control the molecular weight distribution in continuous two-stage nitroxidemediated copolymerizations," Chemical Engineering Journal, vol. 135, no. 1, pp. S242-S246, 2008.

[4] N. Gadish and J. Voldman, "High-throughput positivedielectrophoretic bioparticle microconcentrator," Analytical Chemistry, vol. 78, no. 22, pp. 7870-7876, 2006.

[5] P. I. Frank, P. D. David, L. B. Theodore, and S. L. Adrienne, Fundamentals of Heat and Mass Transfer, John Wiley \& Sons, New York, NY, USA, 2006.

[6] E. L. Cussler, Diffusion Mass Transfer in Fluid Systems, Cambridge University Press, New York, NY, USA, 2009.

[7] N. T. Nguyen and Z. Wu, "Micromixers-a review," Journal of Micromechanics and Microengineering, vol. 15, no. 2, pp. R1R16, 2005.

[8] J. M. Ottino and S. Wiggins, "Introduction:mixing in microfluidics," Philosophical Transactions of the Royal Society A: Mathematical, Physical and Engineering Sciences, vol. 362, no. 1818, pp. 923-935, 2004.
[9] C. S. Lu, J. J. Chen, J. H. Liau, and T. Y. Hsieh, "Flow and concentration analysis inside a microchannel with lightning grooves at two floors," Journal of Biomechatronics Engineering, vol. 2, pp. 13-32, 2009.

[10] R. Miyake, T. S. J. Lammerink, M. Elwenspoek, and J. H. J. Fluitman, "Micro mixer with fast diffusion," in Proceedings of the 6th IEEE International Workshop Micro Electromechanical System (MEMS '93), pp. 248-253, IEEE Computer Society Press, San Diego, Calif, USA, Febuary 1993.

[11] H. Mobius, W. Ehrfeld, V. Hessel, and T. Richter, "Sensor controlled processes in chemical microreactors," in Proceedings of the 8th International Conference on Solid-State Sensors and Actuators (Transducers '95), pp. 775-778, Stockholm, Sweden, June 1995.

[12] F. G. Bessoth, A. J. DeMello, and A. Manz, "Microstructure for efficient continuous flow mixing," Analytical Communications, vol. 36, no. 6, pp. 213-215, 1999.

[13] P. Hinsmann, J. Frank, P. Svasek, M. Harasek, and B. Lendl, "Design, simulation and application of a new micromixing device for time resolved infrared spectroscopy of chemical reactions in solution," Lab Chip, vol. 1, no. 1, pp. 16-21, 2001.

[14] J. S. Maeng, K. Yoo, S. Song, and S. Heu, "Modeling for fluid mixing in passive micromixers using the vortex index," Journal of the Korean Physical Society, vol. 48, no. 5, pp. 902-907, 2006.

[15] C. C. Hong, J. W. Choi, and C. H. Ahn, "A novel in-plane passive microfluidicmixer with modified tesla structures," $L a b$ Chip, vol. 4, no. 2, pp. 109-113, 2004.

[16] M. K. Jeon, J. H. Kim, J. Noh, S. H. Kim, H. G. Park, and S. I. Woo, "Design and characterization of a passive recycle micromixer," Journal of Micromechanics and Microengineering, vol. 15, no. 2, pp. 346-350, 2005.

[17] J. Cha, J. Kim, S. K. Ryu et al., "A highly efficient 3D micromixer using soft PDMS bonding," Journal of Micromechanics and Microengineering, vol. 16, no. 9, pp. 1778-1782, 2006.

[18] A. A. S. Bhagat, E. T. K. Peterson, and I. Papautsky, "A passive planar micromixer with obstructions for mixing at low Reynolds numbers," Journal of Micromechanics and Microengineering, vol. 17, no. 5, pp. 1017-1024, 2007.

[19] M. Koch, D. Chatelain, A. G. R. Evans, and A. Brunnschweiler, "Two simple micromixers based on silicon," Journal of Micromechanics and Microengineering, vol. 8, no. 2, pp. 123126, 1998.

[20] S. Hardt and F. Schonfeld, "Laminar mixing in different interdigital micromixers: II. numerical simulations," AIChE Journal, vol. 49, no. 3, pp. 578-584, 2003.

[21] P. Lob, H. Pennemann, V. Hessel, and Y. Men, "Impact of fluid path geometry and operating parameters on 1/l-dispersion in interdigital micromixers," Chemical Engineering Science, vol. 61, no. 9, pp. 2959-2967, 2006.

[22] S. Bohm, K. Greiner, S. Schlautmann, S. de Vries, and A. van den Berg, "A rapid vortex micromixer for studying high-speed chemical reactions," in Proceedings of the 5th International Conference on Micro Total Analysis Systems (micro-TAS '01), Monterey, Calif, USA, October 2001.

[23] C. H. Lin, C. H. Tsai, and L. M. Fu, "A rapid three-dimensional vortex micromixer utilizing self-rotation effects under low Reynolds number conditions," Journal of Micromechanics and Microengineering, vol. 15, no. 5, pp. 935-943, 2005.

[24] S. Y. Jin, Y. Z. Liu, W. Z. Wang, Z. M. Cao, and H. Koyama, "Numerical evaluation of two-fluid mixing in a swirl micromixer," Journal of Hydrodynamics, vol. 18, no. 5, pp. 542-546, 2006. 
[25] S. Y. Yang, J. L. Lin, and G. B. Lee, " A vortex-type micromixer utilizing pneumatically driven membranesr," Journal of Micromechanics and Microengineering, vol. 19, no. 3, Article ID 035022, 2009.

[26] M. Long, M. A. Sprague, A. A. Grimes, B. D. Rich, and M. Khine, "A simple three-dimensional vortex micromixer," Applied Physics Letters, vol. 94, no. 13, Article ID 133501, 2009.

[27] J. A. Pojman, N. Bessonov, V. Volpert, and M. S. Paley, "Miscible fluids in microgravity (MFMG): a zero-upmass investigation on the international space station," Microgravity Science and Technology, vol. 19, no. 1, pp. 33-41, 2007.

[28] Y. L. Joo and E. S. G. Shaqfeh, "The effects of inertia on the viscoelastic Dean and Taylor-Couette flow instabilities with application to coating flows," Physics of Fluids A, vol. 4, no. 11, pp. 2415-2431, 1992.

[29] D. O. Olagunju, "Inertial effect on the stability of viscoelastic cone-and-plate flow," Journal of Fluid Mechanics, vol. 343, pp. 317-330, 1997.

[30] H. Wang, P. Iovenitti, E. Harvey, and S. Masood, "Numerical investigation of mixing in microchannels with patterned grooves," Journal of Micromechanics and Microengineering, vol. 13, no. 6, pp. 801-808, 2003. 

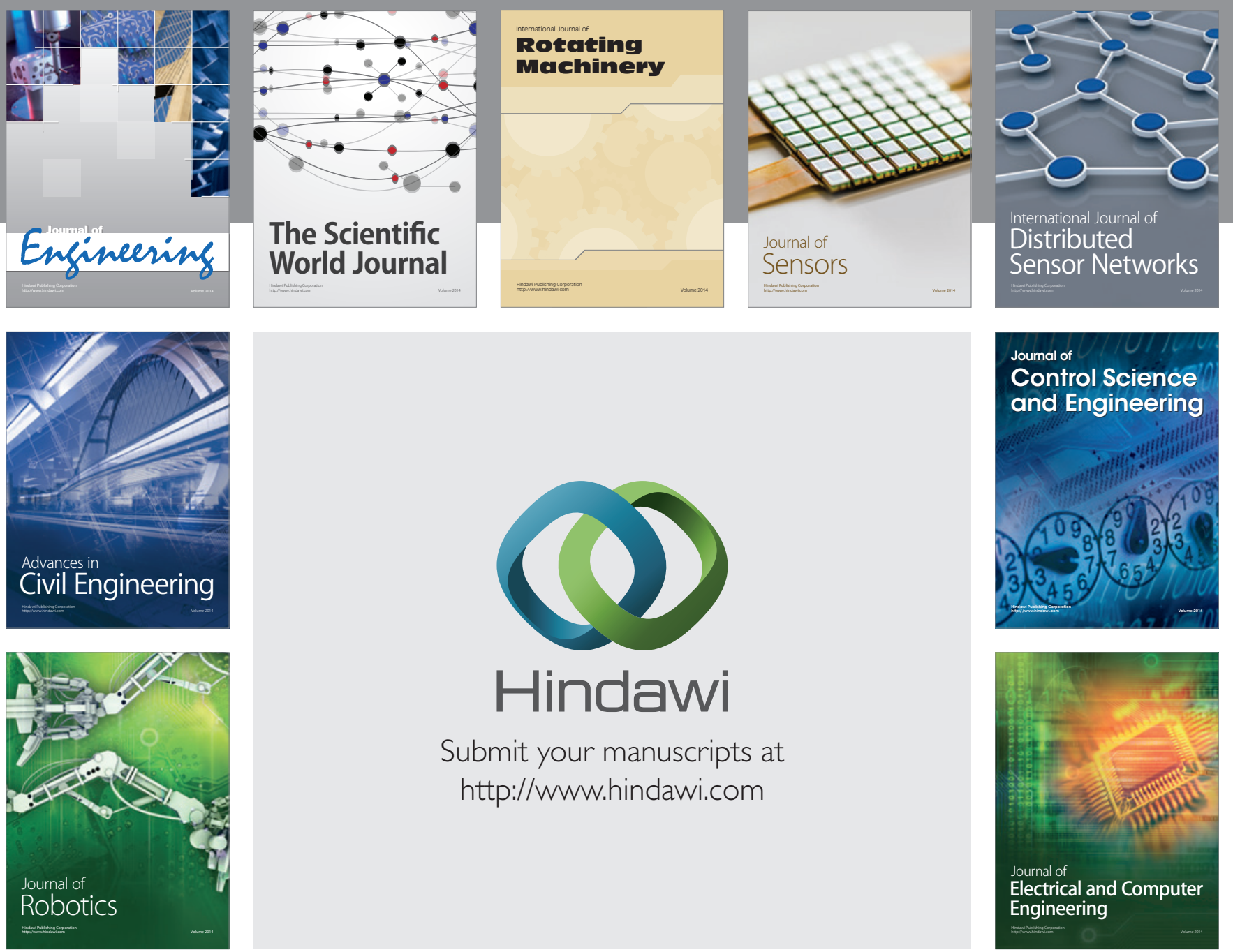

Submit your manuscripts at

http://www.hindawi.com
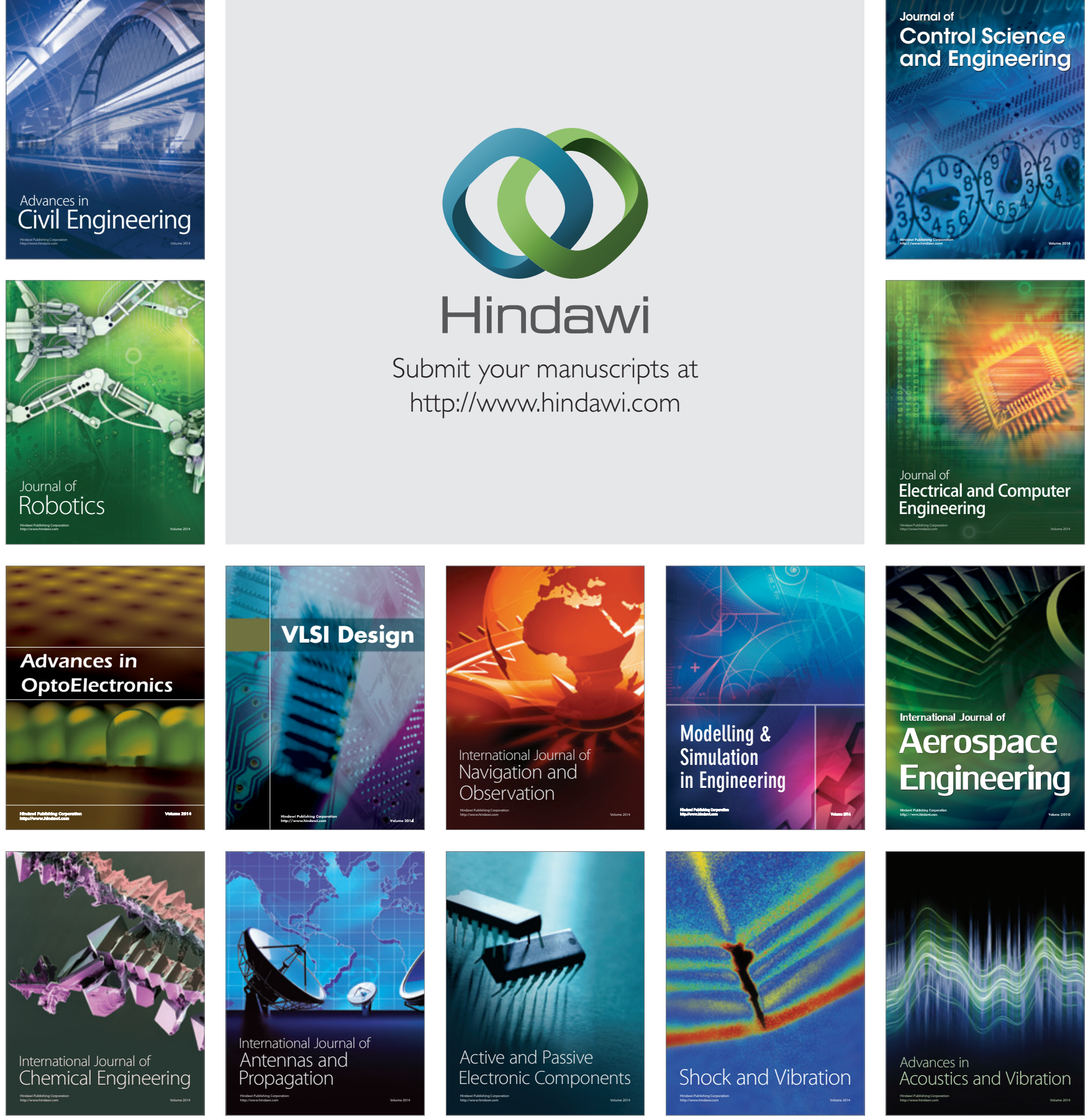